\title{
La educación en Colombia: dinámica del mercado y la globalización
}

Diego Alejandro Rubiano-Aldana*, Harold Beltrán-Jiménez

Licenciado en Pedagogía Infantil. Universidad Distrital Francisco José de Caldas, Bogotá, Colombia. Correo electrónico: diegopedinf@gmail.com

** Maestría en Educación. Profesor, Universidad Distrital Francisco José de Caldas y Universidad InCCA, Bogotá, Colombia. Correo electrónico: hbeltranj@gmail.com

Recibido: 15 de febrero del 2016

Aceptado: 31 de mayo del 2016

Cómo citar este artículo: Rubiano-Aldana, D. A. y Beltrán-Jiménez, H. (2016). La educación en Colombia: dinámica del mercado y la globalización. Cooperativismo \& Desarrollo, 24(109), xx-xx. doi: 10.16925/ co.v24i109.1508

\section{Resumen}

Propósito: presentar una reflexión y construir una interpretación desde la perspectiva del paradigma hermenéutico histórico para generar procesos de comprensión sobre el fenómeno social de la mercantilización de la educación. Descripción: desde 1991, con la reforma constitucional, la educación en Colombia se reconoce como un derecho fundamental. Sin embargo, el proceso de reconocimiento ha estado acompañado por la llegada de las nuevas tecnologías de la información y la comunicación. La educación e-learning, en conjunto con el sistema de franquicias, conformó un modelo de negocio que, en el mercado educativo del país, se presenta como alternativa y estrategia para el desarrollo económico. Punto de vista: esta relación termina incidiendo en la garantía del derecho a la educación al vincular el acceso y la calidad a las posibilidades económicas. Conclusiones: en Colombia se sigue asumiendo el proyecto educativo del país a partir de la coyuntura. La planeación a mediano y largo plazo no hace parte de una política pública coherente porque siempre hay otras prioridades en la asignación presupuestal. Esto, en un país inequitativo como Colombia, implica convertir la educación en un factor que incrementa la desigualdad y no en lo que debería ser: un factor de equidad.

Palabras clave: apertura económica, derecho a la educación, e-learning, franquicias educativas. 


\title{
Education in Colombia: market dynamics and globalization
}

\begin{abstract}
Purpose: To present a reflection and construct an interpretation from the perspective of the historical hermeneutic paradigm in order to generate processes of understanding about the social phenomenon of the commodification of education. Description: Education in Colombia has been recognized as a fundamental right since the constitutional reform of 1991 . However, the recognition process has been accompanied by the advent of new information and communications technologies. E-learning, together with the franchise system, have formed a business model that is presented as an alternative and a strategy for economic development in the country's education market. Viewpoint: This relationship has influenced the guarantee of the right to education by linking access and quality to economic opportunities. Conclusions: In Colombia, the education project of the country continues to depend on circumstances. Planning in the medium and long term is not part of a coherent public policy because there are always other priorities in budget allocation. In an unequal country like Colombia, this has meant turning education into a factor that increases inequality instead of what it should be: a factor of equality.
\end{abstract}

Keywords: economic access, right to education, e-learning, education franchises

\section{A educação na Colômbia: dinâmica do mercado e da globalização}

\section{Resumo}

Propósito: apresentar uma reflexão e construir uma interpretação a partir da perspectiva do paradigma hermenêutico histórico para gerar processos de compreensão sobre o fenômeno social da mercantilização da educação. Descrição: desde 1991, com a reforma constitucional, a educação na Colômbia reconhece-se como um direito fundamental. No entanto, o processo de reconhecimento esteve acompanhado pela chegada das novas tecnologias da informação e da comunicação. A educação e-learning, em conjunto com o sistema de franquias, formou um modelo de negócio que, no mercado educativo do país, apresenta-se como alternativa e estratégia para o desenvolvimento econômico. Ponto de vista: esta relação termina incidindo na garantia do direito à educação ao vincular o acesso e a qualidade às possibilidades econômicas. Conclusões: na Colômbia continua-se assumindo o projeto educativo do país a partir da conjuntura. O planejamento em médio e longo prazo não faz parte de uma política pública coerente porque sempre há outras prioridades na atribuição orçamentária. Isso, num país desigual como a Colômbia, implica transformar a educação num fator que aumenta a desigualdade e não no que deveria ser: um fator de equidade.

Palavras-chave: abertura econômica, direito à educação, e-learning, franquias educativas. 


\section{Introducción}

Este artículo tiene el propósito de presentar una reflexión, construir una interpretación y generar una comprensión sobre el proceso mediante el cual en la educación colombiana se ha consolidado un modelo de negocio en el país. Se pretende abordar un conjunto de antecedentes que involucran aspectos políticos, económicos, jurídicos y legales, insertos en un contexto histórico, que facilitaron que la garantía del derecho a la educación se convirtiera en un servicio cuya prestación no es exclusiva del Estado.

Este artículo se abordará desde una perspectiva hermenéutica que busca aprehender las interpretaciones de la realidad con vistas a la intersubjetividad posible (Ochoa, 2008, p. 13). Se retomarán los aportes de la Escuela de Frankfurt con la intención de plantear la existencia de un conjunto de referencias epistemológicas, que conjugan la historia y su comprensión, tratando de reconstruir la variedad de interpretaciones de textos y hechos sociales que terminan conformando un todo "histórico-hermenéutico", en palabras de Habermas (Vasco, 1990, p. 12).

Para este caso, el esfuerzo hermenéutico y la recapitulación histórica se enfocarán en el análisis del fenómeno de la "mercantilización" de la educación. La noción de mercantilización planteada aquí requiere que se haga uso del concepto de mercancías, entendidas como "cosas que poseen un tipo particular de potencial social, que son discernibles de 'productos', 'objetos', 'bienes', 'artefactos' y otros tipos de cosas (aunque solo sean en ciertos aspectos y desde determinada perspectiva)" (Appadurai, 1991, p. 21). De allí que la educación, al cumplir con estas características, pueda definirse como objeto del mercado, como mercancía.

Gracias al impulso del desarrollo tecnológico, en especial de las Tecnologías de la Información y la Comunicación (TIC), a la necesidad creciente de ampliar la cobertura de la educación, a las demandas sociales que exigen competitividad a través de la educación, y a otros factores, la presencia de la "oferta" educativa ha dinamizado el mercado. Esto requiere que las instituciones educativas se comportan como empresas que buscan aumentar su rentabilidad y productividad en un mercado caracterizado por la extrema competitividad.

Las políticas económicas que promovieron el desarrollo empresarial del país, la apertura económica y la modernización del aparato productivo en los años noventa fueron, precisamente, las mismas que crearon las condiciones para definir la educación como un servicio y, a partir de esto, generar un modelo de negocio. El e-learning, las franquicias de modelos pedagógicos de moda y la masificación de la educación hacen parte del nuevo escenario en el que la educación es una alternativa económica para los empresarios interesados en negocios lucrativos con perspectivas de sostenibilidad.

Para comprender el fenómeno social que facilitó la mercantilización de la educación en Colombia, es necesario entender la influencia de las políticas económicas globales y sus antecedentes históricos, económicos y políticos. El contexto hace parte de la coyuntura en la que se promovió la apertura económica, la modernización del aparato productivo del país y la implementación de un modelo económico basado en la reducción del tamaño del Estado. Se establece un escenario en el que entran en el juego de la economía actores privados que desempeñan roles productivos, que tradicionalmente eran responsabilidad exclusiva del Estado, en sectores de la política social del país. Estas transformaciones se evidencian a partir de la puesta en vigencia de la Constitución Política de 1991 y de las reformas políticas, jurídicas y legales, que intentan cumplir los mandatos de la nueva Constitución, y que tienen un impacto significativo en la transformación de la educación colombiana de finales del siglo xx con las miras puestas en el siglo Xxi. Muchas de estas reformas, sumadas a este nuevo contexto económico, político, jurídico y legal, facilitaron la aparición de nuevas formas, tanto del Estado como de los actores privados, de ofertar el servicio educativo. Esto supuso un impacto enorme en la prestación del servicio y en la garantía del derecho a la educación, en particular, en cuanto a sus indicadores de cobertura y calidad. Habrá una especial atención a fenómenos como el e-learning, y la modalidad de franquicias asociadas a modelos pedagógicos en el país, como evidencias de estas transformaciones.

\section{Antecedentes del problema: apertura económica y liberalización de la economía}

La economía constituye un todo integrado al conjunto del funcionamiento social. En este, las transformaciones culturales, políticas y económicas definen el recorrido histórico de países y comunidades. En el contexto del avance de la Modernidad, estas transformaciones se insertan progresivamente en una dinámica global que define el destino económico de los 
países por las coyunturas y transformaciones de otras naciones. Esto trae consigo varias implicaciones: por un lado, la necesidad de que las economías nacionales generen procesos de apertura que las vinculen con la dinámica global; por otro lado, la necesidad de que el impacto de las dinámicas económicas de algunas potencias económicas y políticas afecte significativamente a la economía global y a las economías locales. En este marco, los países antes llamados "en vías de desarrollo" buscan, con la apertura económica, el camino para el crecimiento económico y el bienestar social. Colombia y los países de la región han apostado por este camino, de modo que sus decisiones y propuestas en esta materia avanzan en consonancia con el proceso de la economía mundial.

A las transformaciones impulsadas por las dinámicas globales se suman las particularidades propias del proceso histórico del país. En Colombia, el impulso de la Modernidad solo ha cobrado fuerza desde finales del siglo xx. La salida de esta "Modernidad postergada" en Colombia (Jaramillo, 2006) ha sido un proceso difícil, discontinuo y lento que tiene un punto de inflexión en la promulgación de la Constitución Política colombiana en 1991. Esta constituye un referente de talante liberal luego de una tradición conservadora dominante en el país. Esta nueva Constitución Política de espíritu liberal tiene implicaciones importantes para la economía y la educación del país, por lo que constituye un referente esencial en esta reflexión.

\section{Contexto económico mundial}

La instauración de un modelo económico basado en un mercado global es un fenómeno que se ha venido asentando a lo largo del siglo xx y que se establece definitivamente durante lo que va del siglo xxi. El modelo se ha venido estabilizando en medio de crisis recurrentes en las que la codependencia entre mercados locales genera dinámicas críticas en el contexto global. La presunta necesidad de facilitar el tránsito de bienes y servicios sin las trabas del proteccionismo local ha animado los acuerdos o tratados de libre comercio. Estos acuerdos multilaterales dinamizan la economía con un balance de pérdidas y ganancias incierto provocado por las asimetrías en las capacidades productivas de los países. Consecuentemente, se generan presiones políticas y sociales por parte de quienes se ven más perjudicados por estas nuevas dinámicas globales.

La idea soñada por Adam Smith (1997, p. 907) se ha venido materializando luego de profundas crisis que han mostrado las glorias y las miserias del liberalismo económico. Desde la tristemente célebre crisis de 1930, que mostró cómo se pasaba de la boyante prosperidad de la Belle Epoque al estupor por la caída de la bolsa - y la imagen del banquero arruinado como emblema-, superada gracias a la doctrina monetarista keynesiana, hasta el reciente estallido de la burbuja inmobiliaria en el 2007, la historia del modelo capitalista ha sido un continuo devenir de épocas de estabilidad y momentos de crisis que no han afectado su inexorable expansión ni la progresiva extinción de modelos alternativos. La creación de la banca central y de la banca multilateral es el corolario de un escenario en el que la batuta de la economía la sostienen el Banco Mundial y el Fondo Monetario Internacional. Estas instituciones son la evidencia de que la idea de producción de la riqueza estaba vinculada a la acumulación del capital: "El desarrollo del comercio y de la industria y de la revolución de los precios, habían hecho al dinero más importante que a los hombres, y la tierra era considerada ahora como fuente de ingreso" (Huberman, 1972, p. 106). La realidad de un capitalismo rampante, basado en la creciente libertad para que la iniciativa privada domine el mercado y en una significativa reducción del poder de intervención de los estados, nos permite visualizar la situación colombiana en este periodo crítico de nuestra historia que es el "entre siglos".

Colombia ha vivido su propio proceso, ha sufrido sus propias crisis y ha construido una historia económica a la sombra de los eventos que impactan la realidad global. El país principalmente agrario del siglo $\mathrm{xx}$ fue abriendo lenta y tímidamente las puertas a una industrialización incompleta. El siglo xx, que fue probablemente para la economía nacional "el siglo del café", se despidió para abrir la puerta a la economía del siglo xxI, dominada por la extracción petrolera como principal eje del crecimiento económico. Este cambio de renglón en la dinámica productiva nacional se dio en las transformaciones políticas, económicas y sociales producidas tras la promulgación de la Constitución Política de 1991: nuevas políticas de apertura económica, modernización del aparato productivo y cambios de paradigma sobre el rol del Estado con la naciente concepción de Estado social de derecho.

\section{Colombia, un Estado social de derecho: políticas de apertura económica}

En Colombia, hay dos periodos de referencia que nos permiten evidenciar los puntos de inflexión que, 
desde la política, la educación y la economía, facilitan la emergencia de lo que hemos dado en llamar un nuevo modelo de negocio para la educación. El primero de ellos es el periodo presidencial de César Gaviria (1990-1994) y el segundo, el que corresponde a los periodos de la primera elección y la primera reelección presidencial de Álvaro Uribe Vélez (20022006 y 2006-2010).

La situación social, económica y política de Colombia, en el momento previo a la llegada de César Gaviria Trujillo al poder en 1990, era convulsionada, para decir lo menos. El panorama general del país se veía enrarecido por el creciente influjo del narcotráfico y el conflicto armado interno, así como por la evidente incapacidad de la clase política para sortear las dificultades y evitar la nefasta influencia del dinero del narcotráfico en la economía y la política misma. Esta situación había enfrentado al país al proceso electoral más accidentado de toda su historia. En él fueron asesinados sucesivamente tres candidatos presidenciales, Luis Carlos Galán, Bernardo Jaramillo y Carlos Pizarro, en menos de un año (entre agosto de 1989 y abril de 1990). El país en su aspecto económico, político y social se encontraba en una grave coyuntura ante el asedio de la violencia guerrillera, paramilitar y mafiosa. Un indicador del atraso y las dificultades del momento era la situación de la industria en Colombia, la cual estaba "rezagada de aquellos sectores que han sido dinámicos a nivel mundial" (Pineda, 1991, p. 496).

La agricultura y la ganadería seguían desempeñando un papel importante en el desarrollo del país, al lado de "un sector industrial fundamentalmente productor de bienes de consumo no durable en la primera instancia, esto es, textiles, confecciones, algunos alimentos, bebidas, tabaco" (Misas, 2001, p. 119). La economía colombiana de entonces, salvo escasas excepciones como el café, el banano y la incipiente extracción minera, estaba pensada para asumir la demanda local y, por tanto, estaba alejada de las dinámicas de globalización que se instauraban en el mundo como parte de una trasformación económica de dimensiones inimaginables para entonces. La economía del país evidenciaba la clara "ausencia de fuerzas competitivas en los mercados internos y la limitada orientación de la oferta hacia los mercados externos", mercados que dificultaban un despertar económico y se situaban "como los grandes responsables de los bajos grados de competitividad y dinamismo de la industria nacional" (Pineda, 1991, p. 500).
El país vivía entonces los efectos de una situación, en la década de los ochenta, marcada por un desequilibrio económico afectado seriamente por el narcotráfico y la violencia. En esta época además se evidenciaba:

Un proceso de estancamiento estructural caracterizado por escasa diversificación productiva (surgen firmas en los sectores existentes, pero los escenarios sociales y se evidenciaba en los altos índices de desempleo, la son pocos los sectores nuevos que se crean), disminución en el ritmo de creación de empresas manufactureras, desindustrialización (pérdida de participación del sector industrial manufacturero en la generación del PIB), pérdida de autonomía tecnológica (Uribe, Vivas y Ortiz, 2010, p. 119).

Pineda (1991, p. 516) indica que en el ocaso de la administración del presidente Virgilio Barco (1986-1990), se centró la atención "en la necesidad de adelantar un proceso de ajuste estructural de la economía nacional para adecuarla a las nuevas realidades del mercado internacional". Este ajuste estaría alineado con las políticas económicas que ya se venían implementando en países industrializados y que anticipaban la necesidad de una apertura económica. Por esto, en un momento bisagra para el desarrollo reciente del país, en 1991, se da una transformación estratégica que tendrá un impacto fundamental en la sociedad, la política y la economía colombiana. En esta coyuntura confluyen, por un lado, la ya mencionada promulgación de la nueva Constitución Política del país y, por el otro, el Plan de Desarrollo de César Gaviria, centrado en la apertura económica y la modernización del aparato productivo. La apertura económica, un modelo que ya había sido implementado con relativo éxito en Chile desde los años setenta, podía entenderse como:

Un conjunto de políticas orientadas a fomentar, entre otras cosas, la entrada de capitales extranjeros y los arreglos del tipo empresa conjunta (joint venture), la rebaja arancelaria y la eliminación de otras trabas a la importación, la negociación de acuerdos comerciales y de integración, el fomento explícito de las exportaciones (Dirven, 2001, p. 78).

La modernización del aparato productivo del país, el otro pilar de la propuesta política de Gaviria, demandaba un lugar distinto para el Estado en la dinamización de la economía. Partiendo de esta premisa, 
junto con la crisis evidente de la sociedad y la política colombiana de entonces, se dio el estado de cosas que llevó a la creación de una Asamblea Nacional Constituyente. Dicha asamblea tenía como tarea derogar la vigente Constitución Política colombiana de 1886 y producir una nueva Carta Magna, la naciente Constitución Política de 1991, que surge como expresión de una necesidad de reestructuración y renovación de los principios de Colombia como EstadoNación. El contexto previo a estas transformaciones es descrito por Pineda (1991, p. 529) como una situación en la que:

El estado no está cumpliendo sus objetivos centrales de desarrollo integral y de justicia social porque su excesivo centralismo, su desbordado formalismo y la ausencia de criterios adecuados en su intervención, han conformado un marco de paternalismo e ineficiencia que le impide cumplir sus finalidades básicas y que, en cambio, obstaculiza y retrasa la acción del resto de la sociedad.

Hernández y Lozano (2001, p. 312) indican que, con el ánimo de fomentar la eficiencia y la competitividad, la llamada apertura económica buscaba, en esencia, mayor influencia del mercado en la asignación de los recursos, para lo cual las reformas enfatizaron la liberación del mercado cambiario, del comercio exterior y del mercado financiero. Esta idea permite entender la forma en que la globalización era una realidad para entonces, lo que hizo que las políticas que se plantearon debieran encaminarse a una internacionalización económica tan inexorable como promisoria.

De allí el empeño de la administración Gaviria en promover tanto la política de apertura económica como la modernización del aparato productivo. Estas políticas contarían con el respaldo del proceso democrático que buscaba fortalecer la figura del Estado a partir del reconocimiento de derechos que se integrarían en el texto constitucional. Como lo señala Pineda (1991, p. 540):

En la fase culminante del proceso de apertura política, el país participó a finales de 1990 en la elección de una Asamblea Nacional Constituyente amplia y democrática. En ella se ha reunido la conciencia nacional en una oportunidad histórica, con el propósito de reestructurar las instituciones, ampliar su base de representatividad y sentar las bases para redefinir las relaciones entre el Estado y la sociedad civil en un nuevo horizonte de paz para la nación.
Una de las definiciones, explícita en la Constitución Política de 1991, de mayor interés para el desarrollo de esta reflexión se encuentra en el título XII ("Del Régimen Económico y de la Hacienda Pública", Capítulo 5 ("De la finalidad social del Estado y de los servicios públicos”). Allí se indica, en el artículo 365, que:

Los servicios públicos son inherentes a la finalidad social del Estado. Es deber del Estado asegurar su prestación eficiente a todos los habitantes del territorio nacional. [...] Los servicios públicos estarán sometidos al régimen jurídico que fije la ley, podrán ser prestados por el Estado, directa o indirectamente, por comunidades organizadas, o por particulares. En todo caso el Estado mantendrá la regulación, el control y la vigilancia de dichos servicios.

El Estado reconoce, entonces, que hay una serie de servicios que deben ser garantizados para la población, y cuando señala que estos "podrán ser prestados por el Estado directa o indirectamente, por comunidades organizadas, o por particulares", abre la posibilidad de que operadores privados presten el servicio en un contexto de mercado abierto. En tal contexto se espera que prime la competitividad, se dinamice la economía, se incentive la inversión extranjera al tiempo que, idealmente, se pueda cumplir con la garantía plena de derechos. Un amplio marco jurídico y legal es creado en los escenarios políticos para promover un proceso de desarrollo estructural en el que el Estado asume un rol descentralizado, reduce su tamaño limitando su burocratización, tan duramente criticada, y se excluye, como un Estado "empresario", de la dinámica del mercado.

LaSubgerencia Culturaldel Banco dela República (2015) señala que la apertura económica, como política económica, es una de las "partes integrales de la ideología política conocida como Neoliberalismo. Según esta, el libre comercio, la descentralización de poder, y la reducción del estado [sic] por medio de la privatización, son algunas de las medidas necesarias para generar el desarrollo nacional". El desarrollo de los principios de la nueva Constitución Política colombiana de 1991 exige la formulación de un nuevo sistema normativo que armonice con el espíritu del texto constitucional y que se articule, además, con el Plan de Desarrollo de la administración Gaviria. Como resultado de este esfuerzo legislativo se promulgan leyes como la Ley 30 de 1992 en la cual se define la Educación Superior como un servicio público 
cultural, inherente a la finalidad social del Estado; la Ley 100 de 1992, en la que se define la seguridad social como un sistema que comprende las obligaciones del Estado y la sociedad (comprende, así, las instituciones y los recursos destinados a garantizar la cobertura de las prestaciones de carácter económico, de salud y servicios complementarios, materia de esta Ley, u otras que se incorporen normativamente en el futuro); y la Ley 115 de 1994, que busca regular el Servicio Público de la Educación que cumple una función social acorde con las necesidades e intereses de las personas, de la familia y de la sociedad. Un Estado menos participativo en las dinámicas del mercado impulsa la iniciativa privada, así como una apertura económica que abre la oportunidad a la globalización progresiva de la economía a través, por ejemplo, de Tratados de Libre Comercio (TLC) y estímulos crecientes para la inversión de capitales extranjeros en Colombia que se puedan ocupar de renglones de la economía y del servicio público que en otros tiempos fueron exclusividad del estado.

\section{La administración Uribe Vélez: entre la profundización del modelo y una nueva economía}

El segundo referente en este ejercicio interpretativo es la administración de Álvaro Uribe Vélez que plasmó, en los planes de desarrollo que presentó respectivamente para los periodos 2002-2006 y 2006-2010, su base económica, política y social. La administración Uribe se basa en los tres principios fundamentales de su política pública: seguridad democrática, confianza inversionista y cohesión social. Estos principios fundamentales buscan la construcción de una equidad social basada en la mejora de la calidad de la educación, un aumento de la cobertura educativa y la reducción de la inversión estatal a favor de una creciente iniciativa privada que asuma muchas de las responsabilidades que deja a un lado el estado, entre otros objetivos. Si bien en 1991 se habían establecido unas políticas que proyectaban un desarrollo económico, la última década del siglo xx se destacó por los problemas sociales que dificultaron el florecimiento económico, no solo en Colombia, sino en países de la región.

Nos referimos aquí a la incapacidad de los distintos gobiernos de hacer cumplir los contratos, las leyes, las normas, los derechos humanos y el conjunto de reglas $\mathrm{y}$ valores necesarios para el buen funcionamiento de los mercados. En el caso de la situación colombiana del momento, la corrupción, el clientelismo, la inseguridad, la pésima administración de justicia, la ingobernabilidad y la pobreza instituida, entre otros, no dejaban tener un buen desempeño económico (Girado, 2007, p. 8).

La oportunidad de crecimiento económico también depende de la inversión extranjera; por tanto, una reestructuración fiscal que favoreciera la incursión en nuevos mercados, la creación de escenarios con regímenes arancelarios especiales como las zonas francas, y la promoción de nuevas inversiones, formó parte de las estrategias que Uribe implementó en el proceso de situar nuevamente al país en la globalización económica. Girado (2007, p. 14) señala que las prioridades del plan eran "sanear las finanzas públicas y restaurar la confianza al sector privado acerca de la situación interna de orden público, creándole expectativas positivas y optimistas a los inversionistas acerca de la seguridad de sus inversiones y rentabilidad de las mismas".

En Uribe-Vélez (2014) se muestra que, como resultado a la implementación de las políticas de su administración, la inversión Extranjera Directa (IED, representada en porcentaje del PIB) alcanzara un 2,3\% en el 2002, 3,1\% en el 2009 y $3 \%$ en el primer trimestre del 2010. Por su parte, la Inversión Extranjera Directa (IED, representada en millones de dólares) llegó a 2100 en el 2002, 7201 en el 2009 y 5013 en el acumulado a 18 de julio de 2010. Además, las exportaciones (en millones de dólares) representaron 11975 en el 2002, 32853 en el 2009 y 16.137 en el acumulado a mayo 2010.

Este estado de cosas permite suponer que una parte de la IED que llegó al país en estos dos periodos de gobierno no solo impactó a sectores tradicionales de la economía nacional, sino que se aprovecharon los nichos productivos que se generan a partir de la progresiva privatización de los servicios públicos (que en otros tiempos eran exclusiva obligación estatal), dentro de los que, por supuesto, tienen especial interés para nuestro análisis los que tienen que ver con la seguridad social y la educación.

\section{Educación e-learning y el sistema de franquicias: dinámica entre la necesidad y la oportunidad}

En los últimos veinte años se consolidó una reestructuración política, jurídica y legal a través de la 
que el Estado asumió el compromiso de garantizar los derechos que en la Constitución Política de 1991 se denominaron fundamentales. La crisis social de la década de los noventa y la necesidad de modernizar el aparato productivo significaron una oportunidad para que los "servicios" de salud y educación, en creciente demanda, fueran cobijados por una política comercial expansiva bajo el liderazgo de la iniciativa privada. Pero no se trata solamente de que los operadores privados presten servicios educativos a través de la creación de instituciones educativas o de la privatización de la operación educativa estatal, sino de la aparición de nuevos nichos de mercado, de nuevas oportunidades de negocio y de nuevos productos y servicios. Dos ejemplos de esos nuevos escenarios a los que se hará especial mención son los programas de educación virtual o a distancia y la aplicación del sistema de franquicias a modelos pedagógicos y educativos que se implementan en instituciones educativas privadas. Estos escenarios novedosos, que traen consigo nuevas oportunidades de negocio, conforman un complejo sistema que pretende contribuir, partiendo de las lógicas del mercado, por supuesto, a la garantía del derecho a la educación.

\section{E-learning: las TIC y los nuevos escenarios de la educación}

El desarrollo científico y tecnológico al que asiste el mundo en la década que finaliza el siglo xx y las décadas que inician el siglo XXI es uno de los procesos más dinámicos y trepidantes a que se hayan presenciado. El campo de las telecomunicaciones, así como su integración con las áreas de la informática y la integración $w e b$, ha tenido un impacto inimaginable en casi todos los ámbitos de la cotidianidad. Las transformaciones a las que se asiste son de una magnitud tal que aún no se dimensiona su impacto en áreas como la educación, el arte y la economía, para mencionar tan solo aquellas que resultan de interés para esta reflexión.

Autores como Benavides (2011) y Verger (2013) abordan esta temática y permiten comprender cómo este nuevo escenario está articulado con las transformaciones sociales, políticas, y, sobre todo, cómo las transformaciones económicas que se han descrito con anterioridad tienen un impacto significativo en las iniciativas con las que se busca garantizar el derecho a la educación. De este modo, nuevas formas de realizar el proceso educativo, y nuevas formas de incorporar el producto de la investigación educativa y pedagógica al quehacer de las instituciones educativas, se abren paso en el país. Todo esto se articula con la creciente necesidad de que la población pueda acceder a la educación a través de las diferentes formas de la oferta que el mercado pone a su disposición. La educación se convierte, de forma inevitable, en un objeto de mercado, un servicio que ofrecen todo tipo de actores como el Estado mismo, operadores privados o las nuevas alianzas público-privadas, como las ilustradas en el siguiente anuncio:

El común esfuerzo de la sED, la Cámara de Comercio de Bogotá, el sENA, las instituciones de Educación Superior (IES) y otras instancias, permitirá ofrecer programas técnicos y tecnológicos de alta calidad y bajo costo, con prácticas opciones de financiación y estrechamente relacionados con las necesidades del mercado laboral y del desarrollo de Bogotá (Centro Virtual de Noticias de la Educación, 2010).

Los nuevos desarrollos tecnológicos, las TIC, dan oportunidad a estrategias en las que las dificultades geográficas, económicas, de uso del tiempo y de infraestructura se superan gracias a proyectos de educación virtual y a distancia. En esta exposición $\mathrm{y}$ argumentación hemos recurrido al término $e$-learning para describir todas las estrategias educativas que hacen uso de las Tic. El término ha adquirido también connotaciones comerciales incuestionables. Para Area y Adell (2009, p. 392), por ejemplo, "el e-learning representa más una etiqueta de marketing que un concepto académico, pero que indudablemente, en la actualidad, ha sido ya asumido como el referente del ámbito de la formación a través de redes de ordenadores".

La globalización ha hecho necesario articular la tecnología a los procesos de formación; es en este contexto en el que la educación e-learning halla su participación en la dinámica del mercado. Las TIC han determinado en gran medida el desarrollo social y económico. Esto supone un cambio para la sociedad, pues:

[Las TIC] constituyen un factor clave en la productividad de los trabajadores, en el tipo de empleo generado, en la evolución de los costes de empresas y, en consecuencia, de su competitividad, así como en sus propios métodos de gestión y organización (Verdeguer y Álvarez, 2001, p. 31). 
En este contexto, Aguadero (1997, p. 84) indica que un reto de la educación frente a sus usuarios es que "tendrá que hacerles competentes en la utilización y manejo de la información y de los conocimientos disponibles; diestros en el manejo de las nuevas tecnologías". Las TIC son en este nuevo escenario, medio, finalidad y contexto del fenómeno educativo.

\section{El modelo de las franquicias educativas}

Otro escenario novedoso para la sociedad globalizada del siglo xxi tiene que ver con las nuevas disposiciones de protección de la propiedad intelectual y la forma de abordar las iniciativas de creación y gestión del conocimiento. No es extraño, entonces, encontrar que modelos pedagógicos generados como innovación educativa sean difundidos e incorporados en nuevos contextos a través de una modalidad específicamente comercial: el sistema de franquicias.

Estesistemaespecificacadadetalledela operación de un negocio en particular: técnicas de producción, símbolos, modos de uso, publicidad, consecución de patentes, estrategias de comercialización y demás aspectos que constituyen jurídicamente la propiedad industrial e intelectual y salvaguardan los intereses del negocio. Para Ortega (2005, p. 73), una franquicia es un:

Convenio en virtud del cual el titular de una empresa que ha desarrollado un establecimiento de comercio dotado de identidad suficiente para distinguirse de todos los demás competidores, otorga una licencia, limitada en el tiempo y el espacio, para que otro comerciante bajo su propio riesgo, replique dicho establecimiento para explotar el mismo ramo de negocios del establecimiento del franquiciante, a cambio de una retribución económica libremente convenida por ambas partes.

En las franquicias existen dos figuras importantes. En primer lugar, el franquiciante o franquiciador, esto es, la persona dueña de los derechos de propiedad intelectual e industrial de una marca o práctica específica. Este tiene la posibilidad de expansión con una baja inversión. En segundo lugar, el franquiciado, es decir, las personas, empresas u organizaciones que tienen el interés de explotar comercialmente la marca promovida por el franquiciante.

Las franquicias se pueden dividir en cuatro categorías dependiendo de la actividad que realicen: la franquicia de producción, la franquicia de distribución, la franquicia de servicios y la franquicia industrial. Colombia e-learning, ciudad e-learning o Catedra sas. Las empresas presentadas anteriormente son ejemplos de la categoría de franquicias de servicios. Esta categoría se caracteriza porque la actividad principal del franquiciador consiste en ofrecer un servicio particular a los consumidores a través de un concepto de mercadeo innovador. Así, Pierre y Rebolledo (2003, p. 28) afirman que: "La franquicia de servicio es la más común en Colombia actualmente".

En coherencia con las políticas comerciales, el sistema de franquicias es una alternativa rentable para los inversionistas y para las empresas, ya que les permite "ampliarse con rapidez así como financiar una parte de ese crecimiento con dinero de terceros" (Raab y Matusky, 2008, p. 14). La expansión de las empresas educativas en el país que hacen uso de este sistema, así como la existencia del e-learning como modelo educativo, consolidan un modelo de negocios que comercializa servicios educativos, eliminando fronteras de espacio y tiempo. EDUPOL, aeiotu de la Fundación Carulla, Kumon, Emprendedores, Young Engineers, Latined, son algunas de las empresas que en la actualidad están en el mercado Colombiano diseñando un portafolio de servicios con una metodología, sistematización y praxis que los diferencia de las instituciones educativas tradicionales. Del mismo modo, el uso de ciertos modelos pedagógicos en instituciones privadas de educación inicial, educación preescolar, educación básica y educación media puede estar guiado por modalidades similares a las franquicias. El uso de estrategias pedagógicas, y del nombre mismo de algunos de estos modelos, acarrea el pago de derechos de propiedad intelectual para respaldar la autenticidad y rigor del modelo. Las escuelas que incorporan la metodología Reggio Emilia o la Enseñanza para la Comprensión son ejemplos de este tipo de modalidad.

\section{Perspectiva de la privatización de la educación}

El hecho de que los países latinoamericanos estén en un contexto en el que se promulgan los derechos es de gran interés para la empresa privada, la cual encuentra en este contexto un campo de generación de negocios. Sumado a esto, hay que resaltar el conjunto de políticas que las naciones han adoptado, principalmente en la última década (CLADE, 2014). Los estudios sobre educación y sociedad de Puiggrós (1999, p. 10) nos llevan a reconocer que hay ciertamente un asunto problemático para los estados neoliberales en lo que respecta a la garantía de los derechos 
fundamentales, en particular la salud y la educación. Por un lado, se esperará siempre que el Estado garantice plenamente estos derechos, pero, para el Estado neoliberal, resulta esencial que parte de esa garantía se ofrezca a través del libre juego del mercado en el que el control estatal termina con frecuencia subordinado a la iniciativa e intereses de los privados, incluso en detrimento de la garantía plena de derechos a la que el Estado le apuesta. La "mano invisible" del mercado, y los presupuestos liberales de la economía en Smith (1997, p. 907), no parecen suficientes para lograr la garantía plena del derecho a la educación. En esta perspectiva hay una reflexión pendiente que permita identificar, por ejemplo, los alcances y limitaciones de la apuesta estatal. Para ello, será necesario revisar el impacto de estas formas de privatización con indicadores puntuales de la garantía del derecho a la educación como la cobertura y la calidad. Basta decir, de entrada, que el solo hecho de que un servicio sea ofertado en condiciones de mercado plantea la pregunta de hasta dónde las limitaciones económicas de los "clientes" limitarán también su acceso a un derecho fundamental y hasta dónde quienes más requieran el servicio sean, paradójicamente, quienes mayores dificultades tengan para adquirirlo. De allí la importancia de revisar este enfoque que permite que la educación sea propuesta como negocio.

La educación, como negocio, se refiere a la manera en la que se concibe esta dentro del modelo económico. Según Petrella (como se cita en Sverdlick, Suazo, Louis, Álvarez y Croso, 2010, p. 67):

En los Estados Unidos y en Canadá (excepto en parte de Quebec) no se hace más que hablar de "mercado de la educación", "negocios de la educación", "mercado de los productos y servicios pedagógicos", "empresas educativas" y "mercado de profesores y alumnos". No es casualidad, sostiene, que el primer mercado mundial de la educación (World Education Market) se haya realizado en Norteamérica, en Vancouver (Canadá), no muy lejos de Seattle. Para este autor, la educación se ha convertido básicamente en un asunto multimedia. El "Mercado mundial de la educación" demostró la existencia de un amplio consenso entre todos los protagonistas públicos y privados presentes: la mercantilización de la educación no deja lugar a duda.

Las posturas políticas de apertura económica coincidieron en la importancia de la creación de mercados en los que la educación se base en la dinámica de la competencia dentro de los sistemas del sector público, lo que tiene el efecto de dividirlos en unidades independientes de negocio (Sverdlick, Suazo, Louis, Álvarez, y Croso, 2010, p. 97). El modelo económico cambia la concepción de la educación, pues "la educación, en efecto, ahora es considerada como una mercancía más, como un servicio" (Gascón y Cepeda, 2008, p. 11). La educación, en las dinámicas de la globalización, tendrá que ser una educación para el mundo, universal, de tal manera que todos puedan contribuir al modelo económico a partir de la oferta de libertad que este les ofrece.

La privatización de la educación termina por dejar en un segundo plano los aspectos del contexto social y cultural, los cuales son sustituidos por una necesidad global. Para sustentar y delimitar claramente lo anterior, puede acudirse a los planteamientos de Acosta y Molano (s. f., p. 3), quienes indican que, a partir de los noventa, se "internacionalizaron un conjunto de discursos y supuestos sobre lo educativo y lo escolar, como parte del proceso de expansión del capitalismo monopolista o globalización y de cambios con incidencia en diversos ámbitos de la cultura, la economía y la política.”

\section{Garantía del derecho a la educación}

El proceso de apertura económica, de globalización, y la descentralización de la economía generaron una dinámica en la que el "desplazamiento progresivo de los costos y la administración de la educación a cargo del Estado, Departamentos y Municipios disminuye los aportes proveyendo las decisiones y los mecanismos para ser entrada con atractivos económicos al manejo del criterio privatizador" (Opciones Pedagógicas, 2002, p. 7). Este hecho nos presenta la oportunidad de rentabilizar la garantía de los derechos a través de modelos empresariales centrados en servicios que fueron por mucho tiempo monopolio estatal. Frente a esta situación, y en el contexto de su relatoría sobre Colombia para la Oficina del Alto Comisionado de las Naciones Unidas para la educación, Katarina Tomasevski (2004, p. 348) afirma que en este estado de cosas la educación "constituye un servicio regulado por el derecho comercial, no por el derecho público de los derechos humanos. El acceso a la educación reflejará entonces el poder adquisitivo de los individuos, no sus derechos". El acceso a la educación, si ha de servir como indicador de la garantía plena de derechos, enfrenta un obstáculo que deslegitima su carácter de servicio. Esto, a su vez, nos 
permite afirmar que, si la educación es un servicio ofrecido por privados, no hay garantía de acceso a poblaciones que tengan bajo poder adquisitivo. Ante estas críticas, uno de los argumentos principales dados a favor de las políticas educativas neoliberales es que el sistema educativo en general se ha caracterizado por no ser eficaz ni equitativo. En este sentido, Puiggrós (1996, p. 91) indica: "De tal afirmación se deduce que la educación pública ha fracasado y se justifican políticas de reducción de la responsabilidad del Estado en la educación, presentadas como la única reforma posible".

La evaluación del cumplimiento de la garantía del derecho a la educación se impone entonces como una necesidad urgente en el propósito de valorar los alcances y limitaciones de la propuesta neoliberal. Este tipo de evaluación ha sido realizado de diferentes formas que van desde el Informe presentado por Tomasevski (2004), pasando por el análisis de la calidad educativa colombiana de Barrera-Osorio, Maldonado y Rodríguez (2012), hasta una serie de informes sobre el tema que aborda diversos aspectos del asunto (Corpoeducación, 2006; Sarmiento, 2011; Delgado, 2014). Para efectos de nuestro análisis, se considerarán de forma general indicadores de cobertura y calidad en relación con los aspectos que son objeto de reflexión de este artículo.

\section{Cobertura}

Uno de los indicadores principales, a partir de la política de apertura económica y de todas las modificaciones realizadas luego de la Constitución Política de 1991, es el de cobertura educativa. La denominación de Estado social de derecho dada a Colombia planteó la necesidad de alfabetizar a la población y garantizar que existieran alternativas para el cumplimiento del derecho a la educación. Los cambios presentados en el orden político nacional contribuyeron enormemente a reducir el rezago educativo existente en Colombia desde los años cincuenta. El incremento de la oferta educativa empezó a ser un indicador de importancia para el gobierno nacional, fenómeno que se explica en la siguiente frase: "Un país más culto logrará tener más rápido una mejor calidad de vida y una economía más estable" (Avendaño y Garzón, 2014, p. 221)

La cobertura educativa se debe entender como la capacidad del Estado para garantizar a los sujetos el acceso al derecho a la educación, y debe medirse por la cantidad de seres humanos que pertenecen al sistema educativo. Desde esta perspectiva, es importante contar con la oferta de las instituciones públicas y privadas que contribuyen a cumplir dicho objeto y generan oportunidades de desarrollo social. Para un Estado que comprende que la educación es el principal activo de la sociedad contemporánea, es importante brindar todas las posibilidades para que sus connacionales se inserten en el sistema educativo; ya que "no sólo es un medio que favorece la movilidad y cohesión social, sino que resulta crucial para impulsar el desarrollo de los países" (Tuirán y Ávila, s. f., p. 1).

El marco político, jurídico y legal existente facilitó el incremento de instituciones educativas (principalmente del sector privado) durante los últimos veinte años, lo cual ayudó a atender la demanda educativa del país. "La política neoliberal en la educación habla de autonomía, descentralización y competitividad desde la visión de mercado y privaticidad, involucrando la pedagogía" (Niño, 2002, p. 38). El informe realizado por el Consejo Privado de Competitividad (CPC) en el 2013 señala que la cobertura y la calidad educativa "es indispensable para reducir la desigualdad, incrementar la productividad laboral y transformar el aparato productivo de un país. Existe amplia evidencia de la correlación entre el nivel educativo de la población y el crecimiento económico de un país". Sin embargo, el Estado no consideró no había una preparación necesaria para el incremento de la cobertura en educación, ni siquiera con el apoyo del sector privado. Esta realidad se empezó a atender a partir del 2002 con la asignación de recursos que permitieron la construcción de megacolegios.

Entre las medidas recientes asumidas por el Gobierno Nacional se destacan, de manera especial, la definición de un esquema de distribución de recursos que incluye la revisión del criterio de población atendida, intentando tener en cuenta de manera adicional la eficiencia y equidad (Ley 715 de 2001 y Decreto 4807 de 2011); el considerable aumento de los recursos que pueden destinarse a la infraestructura educativa (Ley 1450 de 2011); y, por último, la contratación de servicios con entidades privadas ante la insuficiencia de la oferta educativa pública (Leyes 715 de 2001 y 1294 de 2009; Clavijo, 2014).

La cobertura educativa, en el contexto de la modernización del aparato productivo, suma como aliado estratégico al sector privado, el cual contribuye a mejorar la oferta educativa, especialmente en las principales ciudades del país. La dinamización del mercado de la educación satisface, al menos de manera 
parcial, la necesidad del Estado de prestar un servicio que consagra en la Constitución Política como derecho fundamental, incluso asumiendo la dificultad de que este acceso sea limitado por las capacidades económicas de los seres humanos.

El indicador de cobertura debe considerar a la población que no cuenta con los recursos económicos necesarios para acceder a la oferta privada, pues, infortunadamente, existe una relación directa entre el nivel económico de las familias colombianas y la calidad educativa que pueden adquirir. Recordemos que "la cobertura es necesaria pero insuficiente para proteger el derecho a la educación. Se requiere además que aumente con estándares de calidad similares para todas las clases sociales" (García y Quiroz, 2011, p. 138). Las franquicias educativas y la educación $e$-learning pueden concebirse como herramientas que ayudan al aumento de la oferta educativa, al incremento de las cifras en los indicadores de cobertura y, proporcionalmente, en los indicadores de calidad.

En cuanto a la educacion superior, Tuirán y Ávila (s. f., p. 1) resaltan que los países más avanzados tienen niveles de cobertura entre un $60 \%$ o $70 \%$. Incluso los países de desarrollo relativo similar en la región, como Argentina y Chile, cuentan con una cobertura del $68 \%$ y $55 \%$, respectivamente. La superación del rezago en cobertura es imprescindible para lograr una inserción favorable en la emergente economía del conocimiento y reducir las brechas que nos separan de otros países. Esto supone la integración de las modalidades de educación que involucran las Tic, así como una relación de oferta y demanda educativa que sea independiente de las posibilidades económicas de los estudiantes y sus familias.

Avendaño y Garzón (2014, p. 218) nos dicen que América Latina, para 1996, presentó una participación en la producción de la industria electrónica de solo un 3\%. La inversión en ciencia y tecnología en Colombia es $0,13 \%$ del PIB en 1998, lo que muestra una considerable diferencia $(1,9 \%)$ en los países industrializados de la Unión Europea). Igualmente, la capacidad de producción de conocimiento es mínima en Latinoamérica con un $2 \%$ del mercado de tecnología y con un $1,3 \%$ de la producción científica mundial. Por tanto, y ante el crecimiento de la demanda de servicios tecnológicos y su importancia en el desarrollo social, la dinámica de la económica plantea la necesidad de aumentar la cobertura no solo para la disminución del analfabetismo y para la inclusión al sistema educativo, sino también para el aumento de la cobertura de la infraestructura tecnológica y la cobertura de los procesos de alfabetización digital. Las franquicias educativas y la educación e-learning pueden concebirse, así, como herramientas que ayudan al aumento de la oferta educativa, el incremento en los indicadores de cobertura y, proporcionalmente, la garantía del acceso al derecho de la educación.

Gómez (2011, p. 2) señala que "estos sistemas educativos asumen como uno de los componentes básicos la incorporación de las tecnologías de la información y la comunicación (TIC) en el proceso de enseñanza-aprendizaje". Lamentablemente, en la realidad socioeconómica latinoamericana, además de reducir la brecha digital, se debe asumir la responsabilidad de reducir la brecha cognitiva, lo que implica generar procesos de alfabetización.

\section{Calidad}

El concepto de calidad no es propio del campo epistemológico de la educación, ha migrado a diversos escenarios y disciplinas como un concepto trasversal e icónico de la industria y el mercado. Esto quiere decir que el concepto de calidad en la educación aparece como un indicador establecido por el mundo empresarial para medir su competitividad en el contexto y las necesidades a las que responde. Es necesario definir y entender el concepto de calidad, pues, al ser un concepto polisémico, puede sugerir diversas interpretaciones que, en este caso, se deben encaminar a la discusión de la educación como objeto de mercado propuesta en este escrito. La calidad puede ser entendida como una "aptitud para el logro de una misión o propósito, implica la relación con la forma en que cierto producto o servicio se ajusta a un propósito, que usualmente corresponde a las especificaciones del 'cliente"' (González y Espinoza, 2008, p. 250). En una perspectiva más humana, la calidad podría "considerarse un horizonte en la búsqueda del perfeccionamiento humano, según el pensamiento sobre la educación del hombre de Kant" (Niño, 2002, p. 30)

La calidad busca evidenciar la existencia de "autonomía, competitividad, preparación de competencias, rendición de cuentas, excelencia, eficiencia y eficacia que, a la vez, reportan su contingente significativo y práctico al entendimiento de la evaluación y la calidad" (Niño, 2002, p. 29). Todos ellos son factores clave en los procesos, acciones y propuestas emprendidas en el escenario de la educación. Para Gómez (2011, p. 4), este modelo educativo concibe el aprendizaje como una forma en la que se debe otorgar a los estudiantes todos los contenidos concernientes 
a una materia, oficio o disciplina y, además, propiciar escenarios y espacios para la formación de competencias que posibiliten el aprendizaje independiente:

El hecho de pretender que el estudiante conozca el medio, se conozca a sí mismo, conozca los conocimientos y la manera más adecuada para llegar a ellos; implica todo un proceso de aprendizaje autónomo en el que él aprenda a aprender; siendo éste un requisito para la formación por competencias (Salas, s. f., p. 2).

Para profundizar en el entendimiento de esta situación es necesario considerar dos paradigmas educativos que terminan por explicar el sentido y la finalidad de la educación en el país. El primero es el paradigma de la educación que prevalece antes de la promulgación de la Constitución Política de 1991. El segundo es el paradigma de la educación que se desprende del efecto modernizador de la Constitución de 1991. La existencia de estos paradigmas se traduce socialmente en la formulación, aprobación e implementación de políticas que buscan responder a las necesidades de los indicadores educativos del Estado.

El primer paradigma tiene tres premisas fundamentales. La primera de ellas es que la educación es considerada un privilegio, ya que "no sólo está dividida por clases sociales, es decir, los ricos y los pobres envían sus hijos a colegios diferentes, sino que los ricos reciben una educación de mejor calidad que la de los pobres" (García y Quiroz, 2011, p. 138). Lo anterior muestra una relación directamente proporcional entre la calidad educativa y el nivel socioeconómico de las personas. La segunda premisa es que la educación está diseñada para formar una élite intelectual, esto se debe principalmente a que, en el sistema educativo, "los estudiantes con mayor capital social (posición social, conexiones sociales, presencia personal, habilidades de lenguaje, etc.) tienen éxito más fácilmente que los de las clases menos favorecidas" (García y Quiroz, 2011, p. 140). De esta manera, podemos entender la existencia de una organización social en la que los estratos más altos reciben los beneficios de una educación que los prepara para liderar a los estratos más bajos. La última premisa está relacionada con el fracaso educativo, pues no importan los índices de deserción y repitencia escolar que se presenten, lo importante es que la exigencia impuesta a los estudiantes tenga como resultado el éxito de aquellos que logran los requerimientos académicos mínimos que garantizan calidad educativa.
Por su parte, el paradigma moderno de la educación considera que el objetivo de la educación es generar movilidad social, es decir, "la educación emerge como un factor de acumulación de capital humano y movilidad social que representa uno de los elementos claves [sic] del proceso de crecimiento y desarrollo económico" (Iregui, Melo y Ramos, 2006). Otra premisa es la promoción automática reglamentada por el Decreto 230, en el que se indica: "Los establecimientos educativos tienen que garantizar un mínimo de promoción del 95\% de los educandos que finalicen el año escolar en cada uno de los grados" (Presidencia de la república de Colombia, 2002). Esta política implicó que toda una generación se incorporara en esa lógica, la cual trajo serias implicaciones para maestros, estudiantes e instituciones en materia de calidad educativa. A su vez, es una política que contribuyó a un incremento de la desigualdad social.

El paradigma moderno de la educación también implica el importante reto de eliminar la deserción escolar con la formulación de políticas sociales y educativas. Entre ellas encontramos la Política de Cero a Siempre propuesta por el Ministerio de Educación Nacional (MEN, s. f.), la cual busca garantizar una educación gratuita desde los primeros años de la infancia hasta culminar la educación básica y la educación media. Esto tiene que ver directamente con la discusión que ha propuesto este escrito, pues la educación e-learning no escapa a las dificultades que, en general, presenta la calidad de la educación, por el contrario, es un modelo al que hay que mirar con más detenimiento. El CPC (2013) afirma que "en Colombia, al igual que en el resto del mundo, está tomando fuerza la incorporación de las TIC en la educación para ayudar a mejorar la calidad"; razón por la cual no hay que desestimar este recurso. Más bien, hay reclamar al Estado que los procesos de acreditación de alta calidad a los que son sometidos los programas e-learning incluyan elementos que garanticen una educación competente, oportuna para las necesidades de la economía y el mercado laboral colombiano, y que se articule con la realidad sociocultural.

Según el Consejo Nacional de Acreditación (CNA, 2014), los programas académicos e-learning ofertados por las Instituciones de Educación Superior (IES) que deseen ser acreditados con registro de alta calidad deben cumplir los mismos indicadores que un programa académico en la modalidad presencial. Esta afirmación no tiene sentido, pues, si los indicadores que evalúan la modalidad presencial no han logrado garantizar la calidad de ese tipo de educación, 
¿cómo pensar que sí lo lograrán a nivel virtual? La realidad nos muestra que "solo $10 \%$ de las IEs del país (28) cuenta con acreditación de alta calidad y ni siquiera estas se encuentran bien posicionadas a nivel mundial" (CPC, 2013). Por tanto, se deben plantear indicadores de calidad y procesos de evaluación exigentes y minuciosos, pensados en las características especiales de esta modalidad de educación.

Rubio (2003) presenta dos enfoques con los que se busca estandarizar la calidad de los programas de educacion e-learning. Uno de ellos es el enfoque parcial, el cual se centra en la actividad formativa, los materiales de formación, las plataformas tecnológicas y la relación coste-beneficio. Por otro lado, presenta el enfoque global, que parte de los sistemas de evaluación y modelos de calidad estándar como las normas Iso y los sistemas benchmarking (proceso que posibilita a una empresa o institucion compararse con otra que tiene resultados de excelencia con el fin de emularlo). Fainholc (s. f., p. 3) indica que la calidad de la educación e-learning está sujeta a los objetivos que se propone. Adicionalmente, resalta la importancia de tener en cuenta los contextos, cada vez más virtuales, en que se desarrolla esta modalidad, ya que estos implican una paradoja: hay inestabilidad y flexibilidad para una justa valoración. Una clase presencial siempre comienza, y puede comenzar, de diferentes modos; pero, una clase virtual puede "caerse", siendo menos versátil; además, depende de lo que hagan sus estudiantes.

Las franquicias educativas pueden contribuir a mejorar esto, pues, legalmente, este tipo de negocios tiene un minucioso control para todos los procesos que desarrolla. Además, presta la atención y asesoría especializada que requiere la implementación de las prácticas o productos franquiciados. Este sistema puede estandarizar procesos de enseñanza y aprendizaje, ejercer control sobre prácticas y modelos pedagógicos que eleven la calidad educativa. Esto no significa en modo alguno que los modelos pedagógicos en educación deban franquiciarse, ya que eso daría un argumento para fortalecer la concepción de la educación como servicio adjudicado de acuerdo con las posibilidades económicas. Esto mismo es expresado por Pérez (2007, p. 140) al referirse a que las problemáticas del estado hacen que "la educación no se reconozca como un derecho y su realización dependa de la capacidad de pago de las familias". En el contexto de apertura económica y las políticas que se han formulado, sin embargo, no se puede desconocer que este sistema propone estrategias que pueden ser provechosas para la educación en general.

\section{Conclusiones}

La proclamación de los derechos humanos estableció enormes responsabilidades a los gobernantes, pues garantizar su cumplimiento implicó la formulación de normativas que repercutieran realmente en la sociedad. Hacer de la educación un sistema que contribuya a la disminución de la pobreza y la segregación social está ligado a garantizar la escolaridad total de la población, así como a garantizar el acceso a una educación integral, competitiva, igualitaria, justa y en condiciones de calidad que estén sincronizadas con los estándares mundiales. La historia de la economía mundial ha incidido de manera radical en las políticas colombianas. El marco jurídico y legal que dio rumbo a la educación del país delimitó un amplio escenario para el desarrollo económico. Este hecho, lejos de ser negativo para el país, constituye una oportunidad económica. El Estado debe velar por un acceso igualitario y no contribuir a la dinámica en la que la educación está crecientemente desvinculada de la responsabilidad estatal.

La calidad de la educación en Colombia perdió importancia en la década de los noventa, ya que el interés del Estado se concentró en la ampliación de la cobertura. Este hecho tuvo como consecuencia la afectación del nivel de la educación en el país, pues ni el Estado ni las instituciones educativas estaban preparados para atender una amplia demanda de estudiantes. El incremento en la oferta de cupos dificultó el trabajo de los maestros y las maestras, quienes no tenían los suficientes recursos físicos que garantizaran a los estudiantes condiciones básicas para participar de los procesos de enseñanza y aprendizaje. En Colombia se entiende la educación, en términos de competitividad, como estrategia para lograr una formación integral; sin embargo, asumir este modelo trae serias implicaciones que no han sido consideradas de manera concreta.

El sentido y la finalidad de la educación tienen que ver con su democratización y la búsqueda de la mejora de la calidad de vida de los seres humanos. Tiene que ver también con la formación y el comportamiento ético, moral y cultural de la sociedad, lo que da lugar a una educación integral articulada con el conocimiento y el ser. Resolver el asunto de la calidad educativa en Colombia implica una mayor inversión en educación; implica que los grupos de trabajo en las escuelas y las universidades sean más pequeños; exige que se fomente una conciencia de la importancia 
de los efectos de los modelos pedagógicos en los procesos de formación.

Estas dinámicas suponen nuevos retos para la educación, y nos ponen ante la necesidad de que esta se adapte y responda a las necesidades que demanda la sociedad. Las franquicias educativas y las TIC tienen un sistema con estrategias para mejorar la calidad educativa. Las franquicias educativas pueden constituir una forma de privatizar las prácticas, teorías y modelos pedagógicos, ya que están constituidas por un minucioso marco jurídico, político y legal que protege la manera en que son implantadas. En este sentido, el Estado debería dar un paso adelante y construir una franquicia educativa pública, lo que evitaría que este sistema afiance la idea de educación ligada a una dinámica de mercado.

Lamentablemente, en Colombia se sigue haciendo el proyecto educativo del país desde la coyuntura pasajera. La planeación a mediano y largo plazo no hace parte de una política pública coherente, sistemática y estratégica porque siempre hay otras prioridades en la asignación presupuestal. Mientras esta situación continúe, no será posible lograr mejoras sustanciales en los indicadores de calidad de la educación y los logros se seguirán evaluando exclusivamente en términos de la cobertura educativa (indicador que, por otra parte, parece haber tocado techo). La pertinencia de la educación, la calidad de la formación y otros indicadores exigen una revisión del modelo neoliberal que se ha mantenido vigente con su visión de la educación como negocio y con los sesgos que introduce (creer en el aumento de cobertura para optimizar beneficios, en detrimento de la calidad).

No se trata de que los indicadores de calidad y cobertura sean mutuamente excluyentes, sino de pensar en qué modelo económico encuentran sus mejores rendimientos: si en uno que entiende la educación como una iniciativa predominantemente estatal, con incrementos significativos de puntos del pIB para la inversión educativa; o si en uno que buscar fortalecer la iniciativa privada, a sabiendas de que la calidad educativa en el mercado es costosa y que excluye a quienes no disponen de recursos suficientes para invertir en educación. En un país tan inequitativo como Colombia, esto implica convertir la educación en un factor que incrementa la desigualdad, y no en lo que debería ser: un factor de equidad.

\section{Referencias}

Acosta, W. A. y Molano, F. (s. f.). El nuevo orden educativo global y la mercantilización de la escuela pública en bogota 1990-2004. Recuperado de http://bibliotecavirtual.clacso.org.ar/ar/libros/colombia/dcsupn/acostaj_molanoc.pdf

Aguadero, F. (1997). La sociedad de la información: Vivir en el siglo XXI. Madrid: Acento Editorial.

Appadurai, A. (1991). La vida social de las cosas. Perspectiva cultural de las cosas. México: Grijalbo s.A. de c.v.

Area, M., y Adell, J. (2009). eLearning: enseñar y aprender en espacios virtuales. En J. De Pablos Pons, Tecnología Educativa. La formación del profesorado en la era de internet (pp. 391-424). Málaga: Aljibe.

Avendaño, J. y Garzón, R. (2014). La competitividad de Colombia frente a latinoamerica y la educación como factor estrategico para el desarrollo. Revista Entramados - Educación y Sociedad, 1, 211-233.

Barrera-Osorio, F., Maldonado, D. y Rodríguez, C. (2012). Calidad de la educación básica y media en Colombia diagnóstico y propuestas. Bogotá: Universidad de los Andes.

Benavides, G. F. (2011). Mercantilización de la educación y comercio internacional. Revista Expeditio, 8, 19-33.

Centro Virtual de Noticias de la Educación. (Agosto de 2010). A partir de agosto cerca de 50 colegios distritales se convierten en universidades. Recuperado de http://www.mineducacion.gov.co/cvn/1665/w3-article-242500.html

Campaña Latinoamericana por el Derecho a la Educación. [CLADE]. (2014). Tendencias de la privatización de la educación en América Latina y el Caribe. Sao Paulo: CLADE.

Clavijo, S. (3 de marzo de 2014). Recientes avances en la cobertura educativa en Colombia. Comentario Económico del día. Recuperado de http://anif.co/sites/default/ files/uploads/Mar3-14.pdf

Colombia, Congreso Nacional de la República. (29 de diciembre de 1992). Ley 30 de 1992: por la cual se organiza el servicio público de la Educación Superior. Bogotá: Diario Oficial N. ${ }^{\circ} 40.700$, del 29 de diciembre de 1992.

Colombia, Congreso Nacional de la República. (23 de diciembre de 1993). Ley 100 de 1992: por la cual se crea el sistema de seguridad social integral y se dictan otras disposiciones. Bogotá: Diario Oficial N. ${ }^{\circ} 41.148$, del 23 de diciembre de 1993.

Colombia, Congreso Nacional de la República. (8 de febrero 
de 1994). Ley 115 de Febrero 8 de 1994: por la cual se expide la ley general de educación. Bogotá: Diario Oficial 41.214 del 8 de febrero de 1994.

Consejo Nacional de Acreditación. [CNA]. (2014). Lineamientos para la acreditación institucional. Bogotá: Consejo Nacional de Acreditación.

Consejo Privado de Competitividad. (2013). Informe nacional de competitividad 2013-2014. Bogotá: Zetta Comunicadores.

Corpoeducación. (2006). Situación de la educación preescolar, básica, media y superior en Colombia. (Informe para Casa Editorial El Tiempo, Corporación Región, Fundación Corona, Fundación Antonio Restrepo Barco, Plan Internacional, Unicef).

Delgado, M. (2014). La educación básica y media en Colombia: retos en equidad y calidad. Fedesarrollo y Fundación Konrad Adenauer.

Dirven, M. (2001). Complejos productivos, apertura y disolución de cadenas. En CEPAL (Ed.), Apertura Económica y (Des)encadenamientos productivos (pp. 65-110). Santiago de Chile: Naciones Unidas.

Fainholc, B. (s.f.). La calidad en la educación a distancia continúa siendo un tema muy complejo. Red, Revista de Educación a Distancia, 12. Recuperado de http://www. um.es/ead/red/12/fainholc.pdf

García, M., y Quiroz, L. (2011). Apartheid educativo: educación, desigualdad e inmovilidad social en Bogotá. Revista de Economía Institucional, 13(25), 137-162.

Gascón, P. y Cepeda, J. L. (2008). Globalización y mercantilización de la educación: nuevos retos para los Estados y las universidades públicas. Veredas. Revista del pensamiento sociológico, Especial Primer semestre 2008, 7-19.

Girado, F. J. (2007). Balance económico de la administración Uribe. Primer periodo: 2002-2006. Apuntes del CENES (Centro de Estudios Económicos); Escuela de Economía Facultad de Ciencias Económicas y Administrativas Universidad Pedagógica y Tecnológica de Colombia, XXVII(43), 2-25.

Gómez, M. (2011). El modelo educativo basado en competencias en el bachillerato mexicano y el desarrollo de habilidades de informacion. Infoconexión, Revista Chilena de Bibliotecología, 1-28.

González, L. y Espinoza, O. (2008). Calidad de la educación superior: concepto y modelos. Calidad de la Educación, 28, 248-276.

Hernández, A., y Lozano, I. (2001). El estado de las finanzas públicas en Colombia a fines del siglo xx. En G. Misas
(Ed.), Desarrollo económico y social en Colombia: siglo XX (pp. 305-364). Bogotá: Unibiblos.

Huberman, L. (1972). Los bienes terrenales del hombre. Bogota: Editorial la Oveja Negra.

Iregui, A. M., Melo, L. y Ramos, J. (2006). Evaluación y análisis de eficiencia de la educación en Colombia. Recuperado de http://www.banrep.gov.co/docum/ftp/ borra381.pdf

Jaramillo, R. (2006). La postergación de la experiencia de la modernidad en Colombia. Recuperado de http:// www.javeriana.edu.co/narrativa_colombiana/contenido/bibliograf/postergacion.htm

Misas, G. (2001). De la sustitución de importaciones a la apertura económica. La difícil consolidación industrial. En G. Misas (Ed.), Desarrollo Económico y Social en Colombia: siglo xx (pp. 111-145). Bogotá: Unibiblos.

Niño, L. (2002). Dimensiones de la evaluación de la calidad de la educación. Opciones pedagogicas, 25, 27-43.

Ochoa, S. (2008). Habermas: conocimiento e interés. El nuevo estatuto de la razón comprensiva. A Parte Rei. Revista de Filosofía, 1-18.

Opciones Pedagógicas. (2002). Opciones pedagógicas: una tarea pedagógica de 25 números. Revista Opciones Pedagógicas, 3-10.

Ortega, M. (2005). El contrato de franquicia. Revista Foro del Jurista, 26(26), 70-85.

Pérez, L. (2007). La exigibilidad del derecho a la educación a partir del diseño y la ejecución de las políticas públicas educativas. Estudios socio juridicos, 9, 142-165.

Pierre, S. y Rebolledo, C. (2003). La franquicia en colombia: temas, realidades y perspectivas. Bogotá: Editorial Norma.

Pineda, S. (1991). Apertura económica y equidad: los retos de Colombia en la década de los años noventa. En J. O. Melo (Ed.), Colombia hoy: perspectivas hacia el siglo XXI (pp. 477-549). Bogotá: Siglo XXI Editores.

Presidencia de la República de Colombia. (2002). Decreto 230 de 2002: por el cual se dictan normas en materia de currículo, evaluación y promoción de los educandos y evaluación institucional. Bogotá.

Puiggrós, A. (1996). Educación neoliberal y quiebre educativo. Nueva sociedad, 146, 90-101.

Puiggrós, A. (1999). Educación y sociedad en América Latina de fin de siglo: del liberalismo al neoliberalismo pedagógico. Estudios Interdisciplinarios de América Latina y el Caribe, 10(1). Recuperado de http://eial.tau.ac.il/index.php/eial?utm_source=The_Journal_Database 
Raab, S. y Matusky, G. (2002). Franquicias: cómo multiplicar su negocio. México: Limusa.

República de Colombia. (1997). Constitución Política de Colombia. Bogotá: Legis.

Rubio, M. (2003). Enfoques y modelos de evaluacióndele-learning. RevistaElectronicadeInvestigación y Evaluación Educativa, 9(2). Recuperado de http:// www.uv.es/RELIEVE/v9n2/RELIEVEv9n2_1.htm

Salas, W. (s. f.). Formación por competencias en educación superior. Una aproximación conceptual a propósito del caso colombiano. Revista Iberoamericana de Educación, 1-10.

Sarmiento, A. (2011) Situación de la educación en Colombia. Tercera Edición. Educación Compromiso de Todos. Bogotá.

Smith, A. (1997). Investigacion sobre la naturaleza y causa de la riqueza de las naciones. México: Fondo de Cultura Económica.

Subgerencia Cultural del Banco de la República. (2015). Apertura Económica. Recuperado de http:// www.banrepcultural.org/blaavirtual/ayudadetareas/ economia/apertura-economica

Sverdlick, I., Suazo, B., Louis, I., Álvarez, V., y Croso, C. (2010). El derecho a la educación vulnerado, la privatización de la educación en Centroamerica; 1 a ed. Buenos Aires: Fundación Laboratorio de Políticas Públicas.

Tomasevski, K. (2004). Educación: indicadores del derecho a la educación. Revista IIDH, 40, 341-388.

Tuirán, R., y Ávila, J. (s. f.). La educación superior: escenarios y desafíos futuros. Recuperado de http://red-academica.net/observatorio-academico/ wp-content/uploads/2011/05/escenarios.pdf

Uribe, J. I., Vivas, H., y Ortiz, C. H. (2010). Empleo formal y empresas modernas. Perfil de Coyuntura Económica, 16, 117-131.

Uribe Vélez, Á. (2014). Confianza Inversionista. Recuperado de http://www.alvarouribevelez.com.co/ es/content/confianza-inversionista

Vasco, C. E. (1990). Tres estilos de trabajo en las ciencias sociales. Comentarios a propósito del artículo "Conocimiento e Interés", Jürgen Habermas. Bogotá: CINEP.

Verdeguer, E. y Álvarez, L. (2001). La globalización. Madrid: Acento Editorial.

Verger, A. (2013). Políticas de mercado, Estado y universidad: hacia una conceptualización y explicación del fenómeno de la mercantilización de la educación superior. Revista de Educación, 360, 268-291. 